\title{
ESTUDO DAS PROPRIEDADES ESTRUTURAIS E MAGNÉTICAS EM LIGAS HALF-HEUSLER CoMnSb E CuMnSb
}

\author{
STUDY OF STRUCTURAL AND MAGNETIC PROPERTIES IN CoMnSb AND CuMnSb HALF- \\ HEUSLER ALLOYS
}

\author{
Caroline Barlette da Cunha ${ }^{1}$, João Carlos Krause ${ }^{2}$ \\ ${ }^{1}$ Bolsista Pibic/CNPq, Acadêmica de Química Industrial, DCET - URI Campus Santo Ângelo - RS - Brasil. \\ E-mail: kkzooide@yahoo.com.br \\ ${ }^{2}$ Dr. em Ciências - Física Experimental, DCET - URI Campus Santo Ângelo - RS - Brasil. E-mail: krause@ urisan.tche.br \\ *Autor para correspondência: DCET - URI, Prédio 7, Universidade Regional Integrada do Alto Uruguai e Missões, \\ Av. Universidade das Missões, 464 - Santo Ângelo/RS, CEP: 9802-470
}

\begin{abstract}
RESUMO
Desde sua descoberta por Friedrich Heusler, no início do século XX, as ligas Heusler apresentaram interesse científico por caracterizarem-se como ligas ferromagnéticas, constituídas por elementos não magnéticos. Nesse sentido, no presente trabalho, investigou-se a formação de fases, as propriedades estruturais e magnéticas de ligas Heusler $\mathrm{Co}-\mathrm{Mn}-\mathrm{Sb}$ e $\mathrm{Cu}-\mathrm{Mn}-\mathrm{Sb}$ na estequiometria XYZ. No preparo das amostras, estas foram submetidas a tratamentos térmicos que não propiciaram perdas significativas de massa, sendo a maior perda de massa relacionada ao elemento manganês. Das medidas de DRX, pode-se observar a presença de apenas uma fase em ambas as amostras com picos bem definidos representando uma estrutura $\mathrm{C}_{1 \mathrm{~b}}$ com espaço de grupo

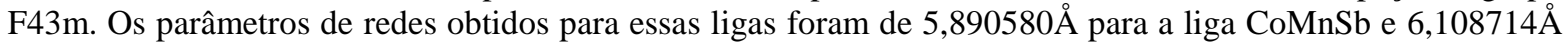
para a liga $\mathrm{CuMnSb}$, valores coerentes com os encontrados nas literaturas para ligas Heusler do tipo halfHeusler. Já nas medidas de histerese, a amostra CoMnSb mostrou-se com uma pequena histerese e com um leve efeito de anisotropia, aspecto que revelou um leve caráter vidro de spin. A amostra $\mathrm{CuMnSb}$ apresentou, também, uma pequena histerese em torno de $0,36 \mathrm{kOe}$ e uma tendência de saturação do ciclo. A partir dos estudos deste trabalho, obteve-se resultados significativos para as duas amostras em estudo, estas apresentando comportamentos característicos de ligas Heusler do tipo half-Heusler.

Palavras-chave: Ligas half-Heusler. DRX. Magnetização.
\end{abstract}

\begin{abstract}
Since its discovery by Friedrich Heusler, at the beginning of the 20TH century, Heusler alloys presented scientific interest because it is describing as ferromagnetic alloys, made of non-magnetic elements. In this sense, the present study was to investigate the formation of phases, structural and magnetic properties of Heusler alloys $\mathrm{Co}-\mathrm{Mn}-\mathrm{Sb}$ and $\mathrm{Cu}-\mathrm{Mn}-\mathrm{Sb}$ in XYZ stoichiometry. In the preparation of these samples were subjected to thermal treatments not provided significant mass loss, being the largest mass loss related to the element manganese. From DRX measures one can observe the presence of only one phase in both samples with well-defined peaks representing a $\mathrm{C} 1 \mathrm{~b}$ structure $\mathrm{F} 43 \mathrm{~m}$ group space. The lattice parameters found to these alloys were 5.890580 $\AA$ for the CoMnSb alloy and $6.108714 \AA$ for the $\mathrm{CuMnSb}$ alloy, values consistent with those found in the literature to half-Heusler alloys. In the CoMnSb sample hysteresis measures, proved with a small hysteresis and with a slight effect of anisotropy, which revealed a slight character spin glass in this sample. For $\mathrm{CuMnSb}$ sample, this also presented a small hysteresis around $0.36 \mathrm{kOe}$ and a saturation trend cycle. From the conclusions of this work if obtained significant results for the two samples under study, these showing characteristic behaviors of half-Heusler alloys.
\end{abstract}

Keywords: Half-Heusler Alloys. DRX. Magnetization. 


\section{INTRODUÇÃO}

Desde sua descoberta por Friedrich Heusler, no início do século XX, as ligas Heusler apresentaram grande interesse científico por caracterizarem-se como ligas ferromagnéticas, constituídas por elementos não magnéticos. No geral, essas ligas são compostos ferromagnéticos formados por átomos não magnéticos, e suas propriedades dependem diretamente dos elementos constituintes, bem como da composição e dos tratamentos térmicos a que são submetidas.

Em razão desses aspectos, as propriedades estruturais, magnéticas e hiperfinas de ligas ternárias tipo Heusler têm sido alvo de intensos estudos nas últimas décadas, tendo em vista as inúmeras propriedades relevantes desses sistemas. As ligas Heusler do tipo $\mathrm{X}_{2} \mathrm{YZ}$ foram estudadas intensivamente, e quase todos os elementos que podem constituí-las já foram objeto de análise; entretanto, algumas ainda carecem de investigação sobre suas propriedades estruturais e magnéticas,

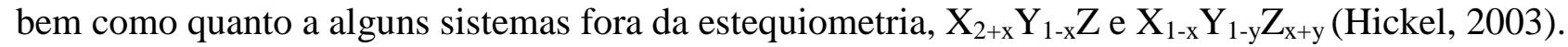

Para essas ligas, há uma gama de possibilidades para as composições, que, além de ter vasta aplicabilidade (Graf et al., 2011), é um dos motivos pelos quais o número de ligas estudadas vem aumentando a cada dia. A combinação de elementos químicos diferentes permite a obtenção de uma grande variedade de ligas que, ao possuírem a mesma estrutura cristalina, possibilitam a comparação dos resultados experimentais, observando os efeitos da alteração da composição. Espera-se que a alteração dos elementos químicos constituintes resulte em sensíveis mudanças nas propriedades magnéticas sem alteração na estrutura do material (Potter, 1931).

Considerando os aspectos apresentados acima, neste trabalho, propõe-se a investigação das propriedades estruturais e magnéticas de ligas half-Heusler tipo XYZ de CoMnSb e CuMnSb, buscando determinar as propriedade estruturais desses sistemas por meio de difração de raios- $\mathrm{X}$ e as propriedades magnéticas por meio de medidas de ciclos de histerese.

\section{REVISÃO DA LITERATURA E FUNDAMENTOS TEÓRICOS}

\section{$2.1 \quad$ Ligas Heusler}

Em 1898, o engenheiro de minas e químico alemão Friedrich Heusler descobriu uma nova classe de compostos intermetálicos ternários, que foi denominado de "ligas Heusler", as quais apresentam interessantes características, tais como a capacidade de se tornarem ferromagnéticas quando submetidas a uma fonte de calor, mesmo sendo compostas de elementos paramagnéticos e diamagnéticos, e, também, de apresentarem um efeito magnetoestrictivo gigante (transição estrutural devida à influência de um campo magnético), elasticidade e um efeito de memória de forma, entre outros.

Existem dois grandes campos de aplicação das ligas Heusler, sendo o mais interessante a spintrônica, que significa investigar os efeitos da magnetorresistência, efeito que ganha destaque por contribuir para a rapidez de leitura e, também, pela miniaturização dos discos rígidos dos computadores. O outro grande campo é o de efeito de memória de forma, que se baseia na redistribuição dos domínios martensíticos, induzidos por um campo magnético na amostra.

Como se sabe, essas ligas apresentam subdivisões, conforme a sua estequiometria, denominadas de (Graf et al., 2011):

- Full-Heusler (Heusler completamente ordenada): os elementos apresentam-se em estequiometria $\mathrm{X}_{2} \mathrm{YZ}$, possuindo uma estrutura L2 ${ }_{1}$ (Figura 1) e espaço de grupo Fm3m. 


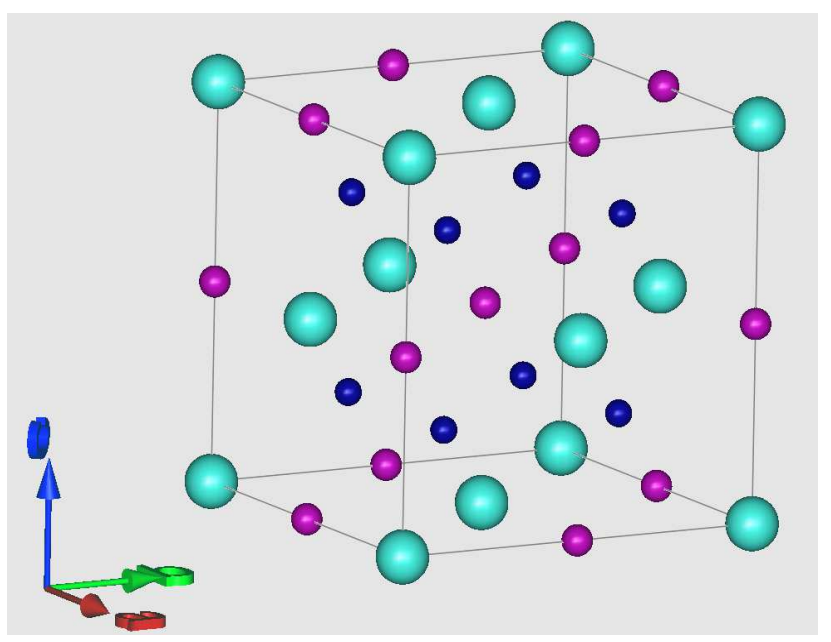

Figura 1: Estrutura Heusler do tipo L2 1

- Half-Heusler (Heusler semiordenadas): os elementos apresentam-se em estequiometria XYZ, possuem como estrutura $\mathrm{C}_{\mathrm{b}}$, sendo esse tipo de estrutura também $\mathrm{CFC}$, mas pertencente ao grupo F43m, com parâmetro de rede que diferencia-se ligeiramente do parâmetro de rede de uma liga Heusler do tipo full-Heusler.

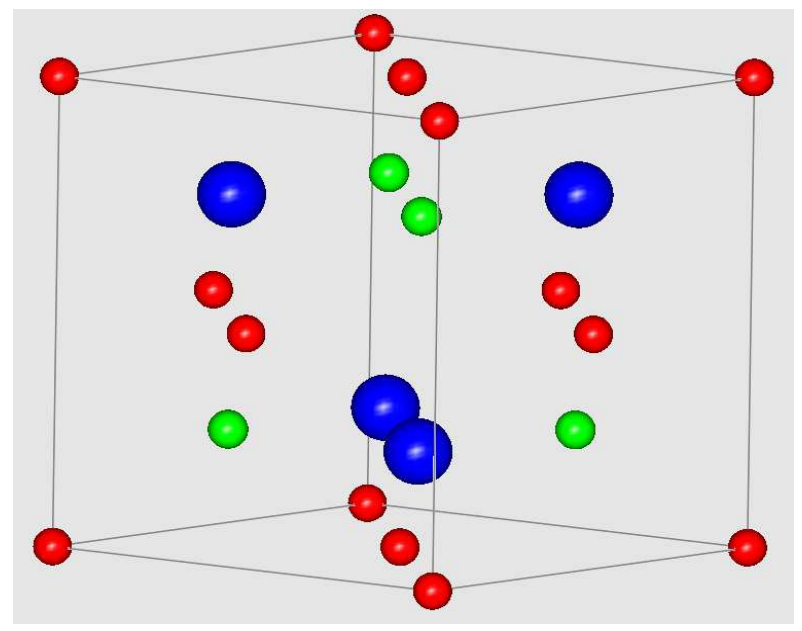

Figura 2: Estrutura Heusler do tipo $\mathrm{Cl}_{\mathrm{b}}$.

Esses compostos são sempre preparados a partir da mistura de três elementos, sendo, em geral, metais de transição, cuja composição estequiométrica é citada acima, onde os elementos $\mathrm{X}, \mathrm{Y}, \mathrm{Z}$ representam:

X: Um metal nobre ou de transição $3 \mathrm{~d}$, 4d ou $5 \mathrm{~d}$ com a camada d externa mais que meio cheia.

Y: Um metal de transição $3 \mathrm{~d}, 4 \mathrm{~d}$ ou $5 \mathrm{~d}$ com a camada d externa menos que meio cheia.

Z: Um metal de transição tipo s-p, ou seja, metais com a camada d externa totalmente incompleta ou completa (Webster et al., 1988; Graf et al., 2011).

A figura a seguir mostra os respectivos elementos da tabela periódica que podem compor esses sítios X, Y e Z. 


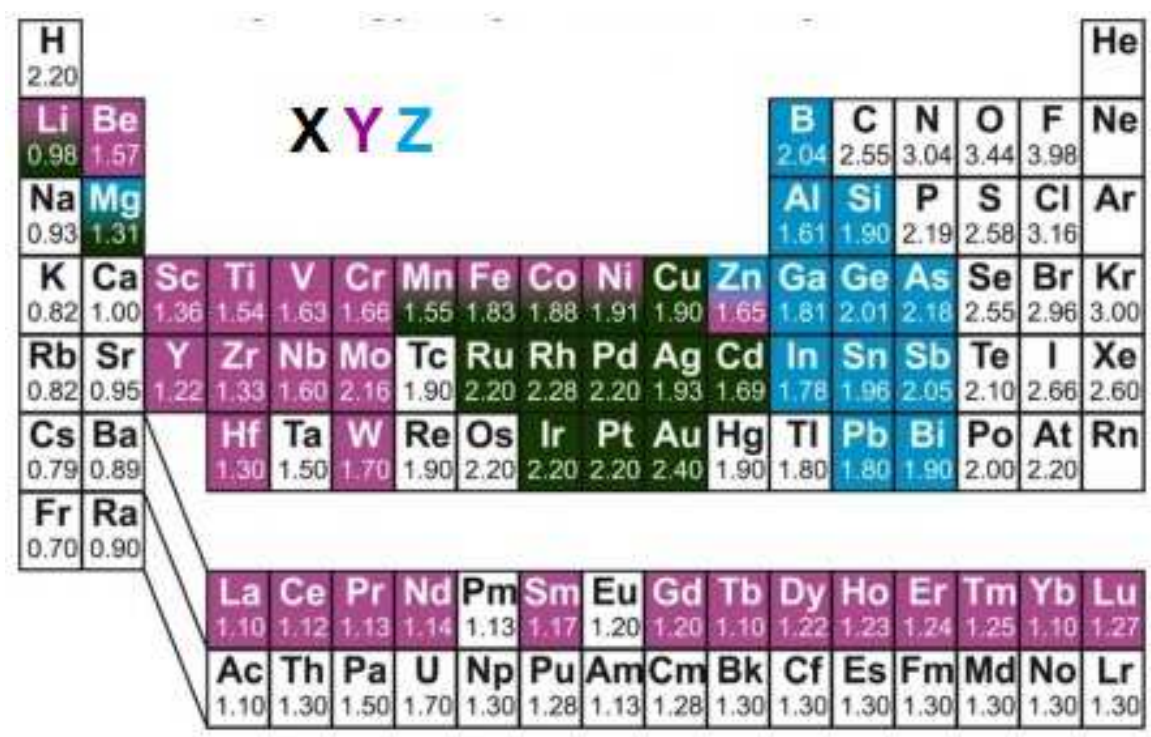

Figura 3: Elementos que podem compor as ligas Heusler na tabela periódica. Fonte: Graf et al., 2010.

\section{MATERIAIS E METÓDOS}

Para obtenção das amostras das ligas em estudo, foram pesados os elementos com alto grau de pureza ( $\geq 99,5 \%)$ constituintes de cada amostra nas estequiometrias previamente definidas (Tabela 1) e fundidas em um forno a arco voltaico, sob atmosfera inerte de argônio. Após a fusão, as amostras foram encapsuladas em tubos de quartzo e submetidas a tratamentos térmicos em um forno tipo mufla por 1 dia a $660^{\circ} \mathrm{C}$. Ao final, foi realizado um resfriamento rápido (quenching) em água gelada, seguido de um recozimento a $240^{\circ} \mathrm{C}$ por 3 dias. As fusões e os tratamentos térmicos foram realizados no Laboratório de Materiais Magnéticos (LAMM ) situado na URI, campus Santo Ângelo.

Após os tratamentos térmicos, as amostras foram preparadas na forma de pó e encaminhadas para medidas de difração de raios-x, realizadas no Instituto de Física da UFRGS, e seus difratogramas foram obtidos e analisados por meio do método de Rietveld, com o auxílio do software WinPlotR®.

Foi prevista a perda de massa de $\mathrm{Mn}$, sendo feito um ajuste de $10 \%$ a mais na massa desse composto, com o objetivo de não ser perdida a estequiometria pretendida para as ligas em estudos, isso em razão de o ponto de fusão do Mn estar muito abaixo dos demais componentes da liga.

Depois das análises de difração de raios-X, as amostras foram submetidas a medidas de magnetometria, as quais foram feitas no Centro de Desenvolvimento Tecnológico Nuclear (CDTN) em Minas Gerais, com a colaboração do Dr. José Domingos Ardisson. Nesse processo, pôde-se obter resultados significativos para as amostras, no que se refere à histerese $\mathrm{M}(\mathrm{H})$ das ligas em estudo, os quais podem ser verificados na próxima seção.

\section{RESULTADOS}

Foram estudadas duas amostras, CoMnSb e CuMnSb, cujas composições estão expressas na Tabela 1.

Tabela 1: Estequiometrias pretendidas para cada amostra

\begin{tabular}{lllll}
\hline Amostra & $\mathrm{Co}$ & $\mathrm{Cu}$ & $\mathrm{Mn}$ & $\mathrm{Sb}$ \\
\hline CoMnSb & $33,3 \%$ & - & $33,3 \%$ & $33,3 \%$ \\
CuMnSb & - & $33,3 \%$ & $33,3 \%$ & $33,3 \%$ \\
\hline
\end{tabular}


Para início das análises, inicialmente, foram simulados difratogramas de raios-x para uma estrutura $\mathrm{C}_{1 \mathrm{~b}}(\mathrm{~F} 43 \mathrm{M})$, a qual pode ser observada a seguir.

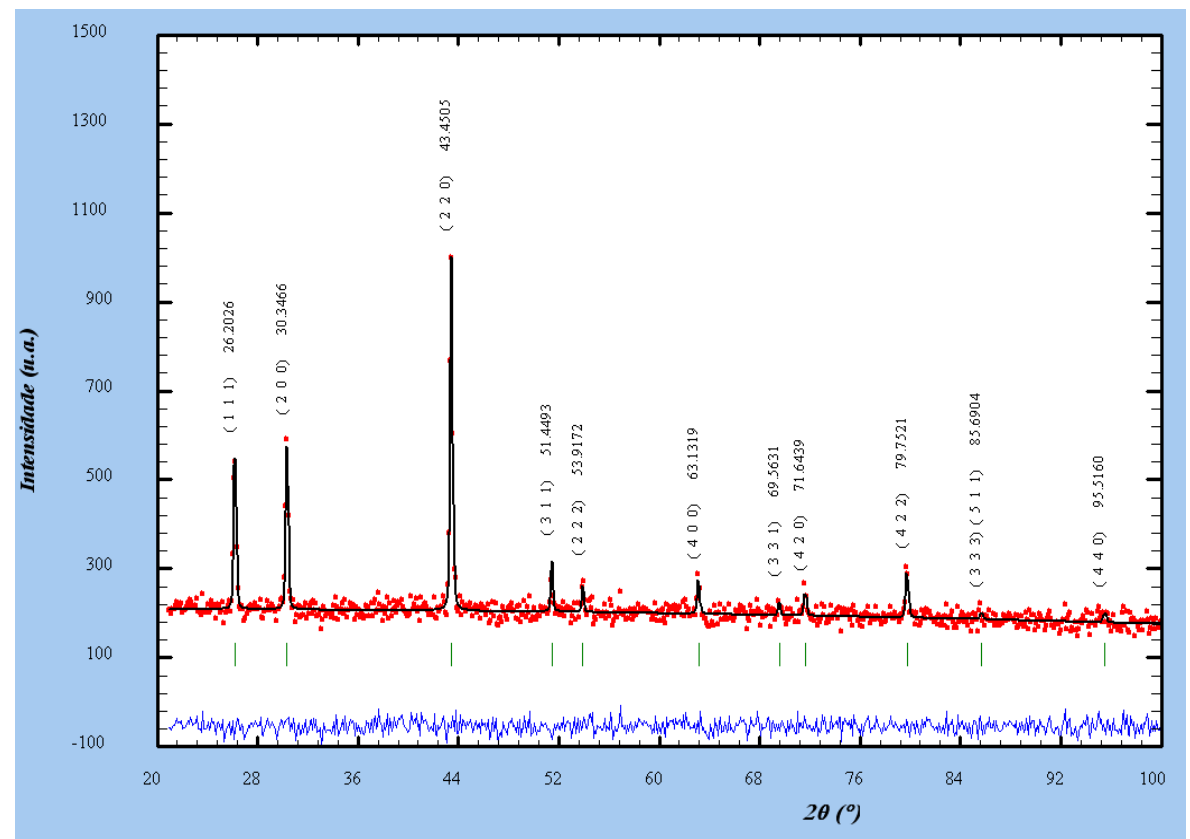

Figura 4: Difratograma de raios-x simulado para uma liga half-Heusler CoMnSb

A Figura 4 corresponde a um difratograma DRX simulado de uma liga CoMnSb com estrutura tipo $\mathrm{C}_{1 \mathrm{~b}}(\mathrm{~F} 43 \mathrm{~m})$, que corresponde a um cristal como o apresentado na Figura 2.

Após terem sido obtidos para cada uma das amostras, os difratogramas foram analisados e ajustados com o WinPLotR $®$ e podem ser visualizados nas figuras a seguir.

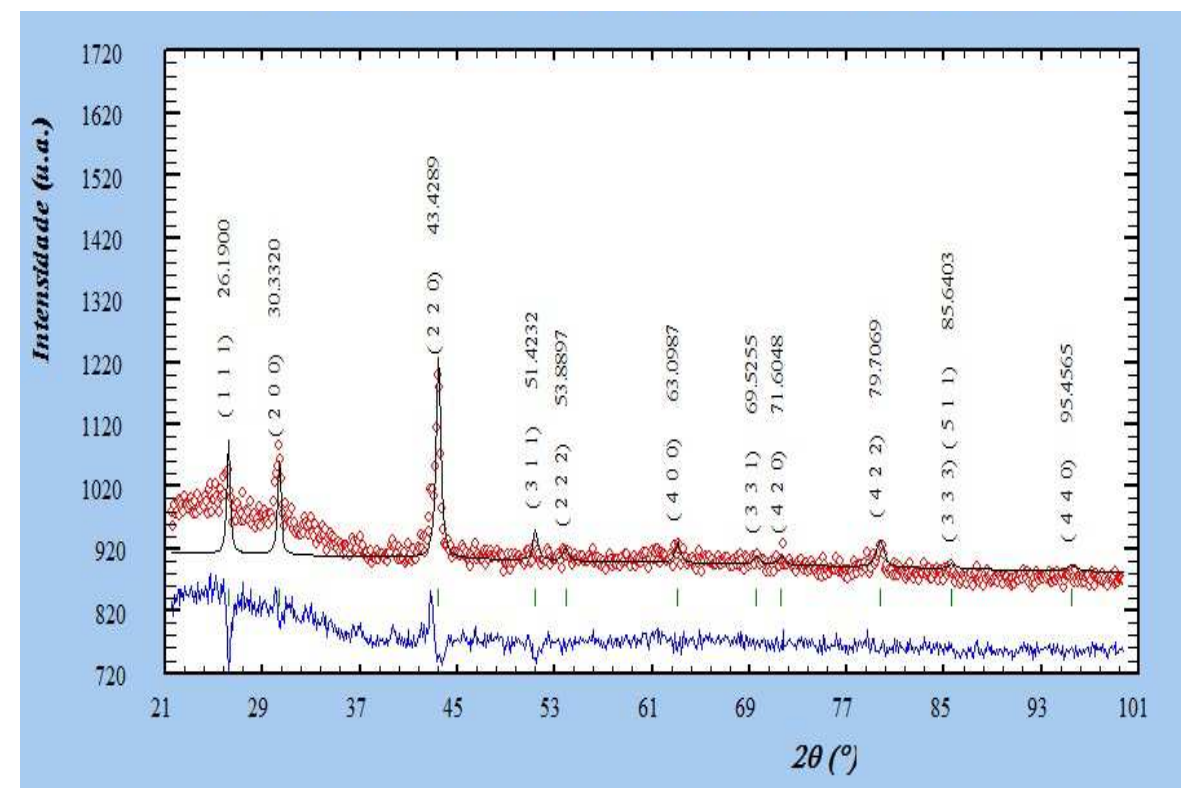

Figura 5: Difratograma de raios-X para a amostra CoMnSb analisado com o WinPlotR ${ }^{\circledR}$. A linha vermelha representa os dados experimentais; a linha preta, o difratograma calculado; a linha azul é a diferença entre o experimental e o calculado; e os traços verdes são as posições dos picos (hkl), índices de Müller 


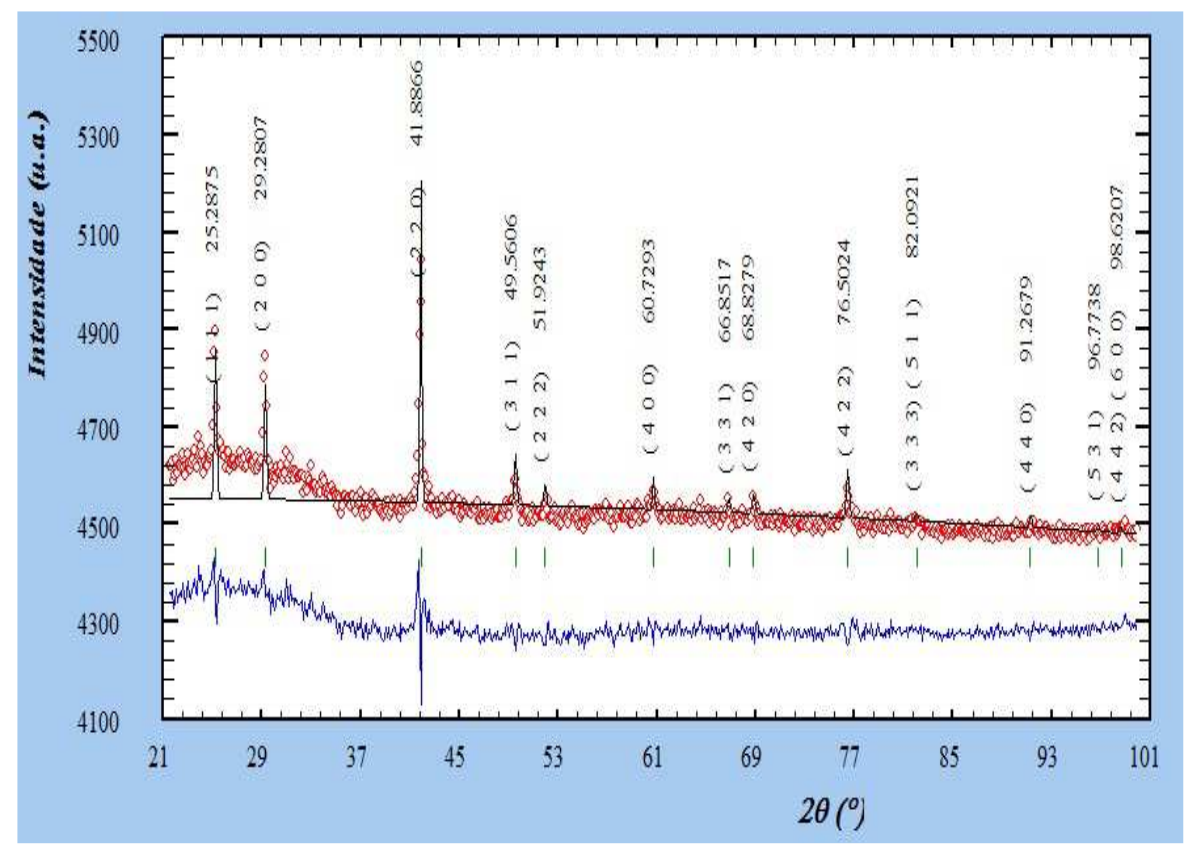

Figura 6: Difratograma de raios-x para a amostra CuMnSb analisado com o WinPlotR®. A linha vermelha refere-se aos dados experimentais, a linha preta representa o difratograma calculado, a linha azul diz respeito à diferença entre o experimental e o calculado e os traços verdes são as posições dos picos (hkl), índices de Müller

Por meio da análise dos raios-x, observou-se a formação de uma única fase em ambas as amostras, sendo essas do tipo $\mathrm{C}_{1 \mathrm{~b}}$, tipicamente de uma liga Heusler do tipo half-Heusler, com espaço de grupo F43m. Na figuras de difração de raios-X, pode-se observar, também, os respectivos hkls para cada um dos picos dos difratogramas.

Com os resultados obtidos com auxílio do software WinPlotR®, pôde-se avaliar os parâmetros de rede dessas amostras, sendo esses característicos a uma liga de Heusler. Os resultados obtidos para os parâmetros de rede estão sistematizados na Tabela 2.

Tabela 2: Parâmetros de rede das amostras CoMnSb e CuMnSb

\begin{tabular}{cccc}
\hline Amostras & $\mathrm{a}(\AA)$ & $\mathrm{b}(\AA)$ & $\mathrm{c}(\AA)$ \\
\hline $\mathrm{CoMnSb}$ & 5.890580 & 5,890580 & 5,890580 \\
& & & \\
$\mathrm{CuMnSb}$ & 6,108714 & 6,108714 & 6,108714 \\
\hline
\end{tabular}

$\mathrm{Na}$ magnetometria, foram investigadas algumas das propriedades magnéticas das ligas em estudos, tendo o principal objetivo da magnetometria sido a medida da magnetização (intrínseca ou induzida) de um material. Com isso, nas medidas de histerese (Figura 7), observou-se que a amostra $\mathrm{CoMnSb}$ apresentou um valor pequeno de histerese e com uma leve tendência à não saturação do ciclo. Outro ponto relevante observado na medida de histerese da amostra foi que esta apresentou um caráter anisotrópico, ou seja, a preferência que um material magnético dispõe de se magnetizar numa determinada direção (Fischer et al., 1991). Essa anisotropia pode revelar um leve caráter vidro de spin nessa amostra, como pode ser observado pelo quadro em destaque no gráfico das medidas de histerese (Figura 7).

Já para a amostra CuMnSb, pôde-se observar a presença de uma pequena histerese em torno de $0,36 \mathrm{H}(\mathrm{kOe})$, mas bem maior do que aquela apresentada pela liga CoMnSb, e a saturação do ciclo. 


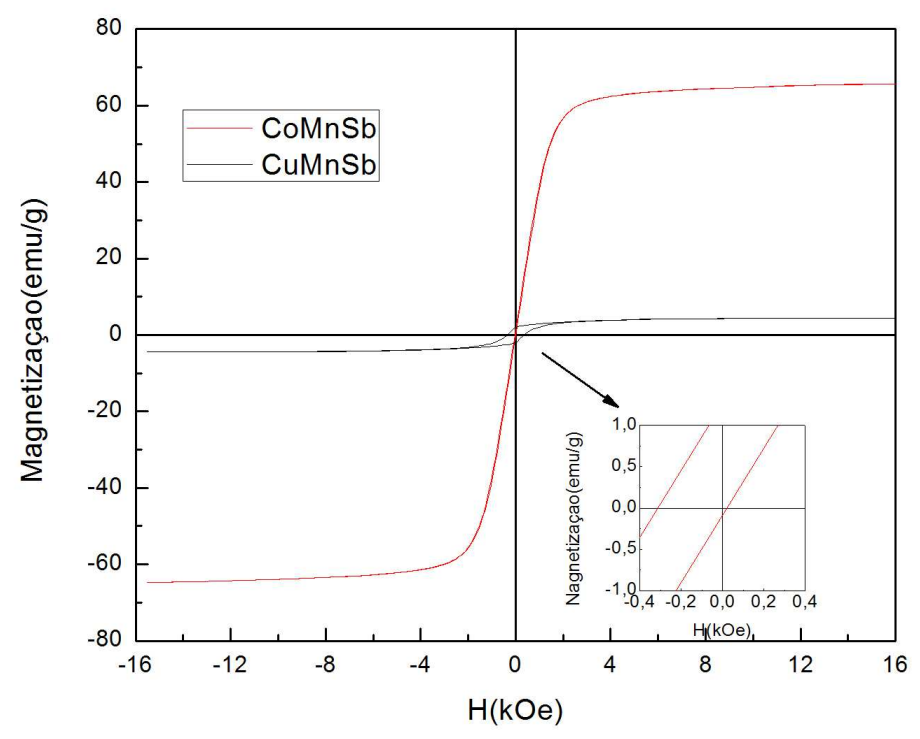

Figura 7: Medidas de histerese das amostras em estudo

Com os valores das massas dos compostos em estudo, pôde-se saber quanto se tem em massa em 1 g de cada elemento e quantos átomos há em cada amostra, para, assim, realizar o cálculo da magnetização por átomo da amostra, podendo os valores do número de átomos em cada amostra ser visualizados na Tabela 3 .

Tabela 3: Valores calculados de cada composto referentes à sua massa e ao seu valor de átomo em $1 \mathrm{~g}$ de amostra

Amostras Massa calculada $(\mathbf{g})$ $\mathrm{N}^{0}$ de átomos calculados

\begin{tabular}{ccc} 
& & (átomos) \\
\hline $\mathbf{C o}$ & 0,22739 & $2,323 \times 10^{21}$ \\
$\mathbf{M n}$ & 0,30291 & $3,319 \times 10^{21}$ \\
$\mathbf{S b}$ & 0,46978 & $2,322 \times 10^{21}$ \\
\hline $\mathbf{C u}$ & 0,23526 & $2,229 \times 10^{21}$ \\
$\mathbf{M n}$ & 0,29879 & $3,274 \times 10^{21}$ \\
$\mathbf{S b}$ & 0,460706 & $2,277 \times 10^{21}$ \\
\hline
\end{tabular}

Com base nesses valores, obteve-se o momento magnético médio de cada amostra e o momento magnético considerando apenas os átomos de manganês magnéticos, pois esse é o único composto com alguma propriedade magnética presente na amostra. Os valores obtidos podem ser visualizados na Tabela 4.

Tabela 4: Valores em Mb/átomos

\begin{tabular}{ccc}
\hline & & $\boldsymbol{\mu}_{\mathrm{B}} /$ átomo \\
\hline \multirow{2}{*}{ CoMnSb } & Todos os átomos & 0,888 \\
& Apenas átomos de Mn & 2,131 \\
\hline \multirow{2}{*}{ CuMnSB } & Todos os átomos & 0,060 \\
& Apenas átomos de Mn & 0,144 \\
\hline
\end{tabular}

\section{DISCUSSÃO DOS RESULTADOS}

Quanto às medidas de difração de raio-x (DRX), observou-se que, quando comparadas a estudos anteriores, essas amostras possuem propriedades estruturais muito semelhantes, tanto na análise de 
seus difratogramas quanto nos valores obtidos para os parâmetros de rede, sendo coerentes com estudos experimentais anteriores, que indicam que os parâmetros de rede das ligas CoMnSb são da

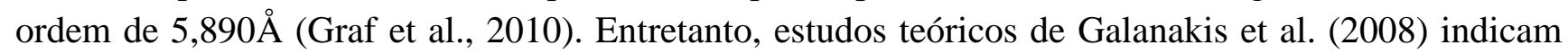
que o parâmetro de rede desses compostos são da ordem de $5,73 \AA$, não concordando com o presente trabalho e com o estudo de Graf citado anteriormente. Já o parâmetro de rede da amostra CuMnSb mostra-se coerente com estudos precedentes, indicando um parâmetro de rede de $6,1044 \AA$ (Podgornykh et al., 2007).

Outro ponto relevante relacionado ao difratograma dessas amostras é que os picos de superestruturas são facilmente observados, nas posições (111), (311), (331), referentes à estrutura C1b, que é característica a uma liga Heusler do tipo half-Heusler (Podgornykh et al., 2007).

Quanto às posições dos elementos na rede cristalina, pode-se afirmar, com base em trabalhos anteriores, que o elemento $\mathrm{Cu}$ ocupa uma das subredes FCC; já os elementos Mn e Sb ocupam duas outras subredes respectivamente, e a quarta subrede permanece desocupada (Tobol et al., 2000).

Já para as medidas de magnetização, obteve-se resultados significativos com relação à magnetização desses compostos; no entanto, não foram encontrados na literatura resultados para comparação. Os valores dos momentos magnéticos obtidos são consideráveis, visto que os átomos que compõem cada amostra são, em sua maioria, não magnéticos, corroborando o caráter magnético apresentado pelas ligas Heusler, ou seja, apresentar magnetização, mesmo sendo constituída por átomos não magnéticos. Outro aspecto relevante observado foi a leve anisotropia na liga CoMnSb, característica já observada em ligas Ni-Co-Mn-Sb (Nayak et al., 2010).

\section{CONCLUSÃO}

Com base na análise dos resultados, conclui-se a possibilidade de formação de uma liga Heusler do tipo half-Heusler, tanto para amostra CoMnSb como para a $\mathrm{CuMnSb}$, tendo os difratogramas apresentado uma única fase com parâmetros de rede coerentes com os observados na literatura. Pôdese constatar, também, que os tratamentos térmicos não ocasionaram perdas muito significativas de massa.

Para os resultados obtidos das medidas de histerese, concluiu-se que a amostra CoMnSb apresentou-se com uma pequena histerese e com um leve efeito de anisotropia, somente observado com a ampliação da medida, revelando um leve caráter vidro de spin nesta amostra. Tal fato evidencia a possibilidade de aplicação das ligas Heusler em spintrônica, devido à presença desse caráter. Por fim, a amostra CuMnSb também se apresentou com uma pequena histerese, em torno de 0,36 kOe, e pôde-se observar que o ciclo saturou completamente.

\section{REFERÊNCIAS}

FISCHER, K. H.; HERTZ, J. A. Spin glasses. New York: Cambridge University Press, 1991.

GALANAKIS, I. et al. Magnetic phase transition in half-metallic CoMnS and NiMnSb semi-Heusler alloys upon $\mathrm{Cu}$ doping: First-principles calculations. Physical Review B, v. 77, p. 214417-1/ 214417$5,2008$.

GRAF, T. et al. Phase separation in the quaternary Heusler compound CoTi(1x)MnxSb - A reduction in the thermal conductivity for thermoelectric applications. Elsevier, v. 63, p. 1216-1219, 2010. 
GRAF, T.; PARKIN, S. S. P.; FELSER, C. Heusler compounds - A material class with exceptional properties. IEEE Transactions on Magnetics, v. 47, n. 2, feb. 2011.

WESTBROOK, J. H., FLEISCHER, R. L. Intermetallic Compounds: Magnetic, Eletrical and Properties and Aplications of Intermetallic Compounds, John Wiley \& Sons, Inc., New York, 2000.

NAYAK, A. K.; SURESH, K. G.; NIGAM, A. K. Phase coexistence induced by cooling across the first order transition in Ni-Co-Mn-Sb shape memory alloy. Journal of Applied Physics, v. 108, n. 6, p. 063915-063915-7, 2010.

PODGORNYKH, S. M. et al. Heat capacity of Heusler alloys: Ferromagnetic Ni2MnSb, Ni2MnSn, $\mathrm{NiMnSb}$ and antiferromagnetic CuMnSb. Journal of Magnetism and Magnetic Materials, v. 311, p. 530-534, 2007.

POTTER, H. H. Some magnetic alloys and their properties. Philosophical Magazine, v. 12, n. 76, p. 255-264, 1931.

TOBOLA, J.; PIERRE, J. Electronic phase diagram of the XTZ ( $\mathrm{X}=\mathrm{Fe}, \mathrm{Co}, \mathrm{Ni}$; T=Ti, V, Zr, Nb, Mn; $\mathrm{Z}=\mathrm{Sn}, \mathrm{Sb}$ ) semi-Heusler compounds. Journal of Alloys and Compounds, v. 296, p. 243-252, 2000.

WEBSTER, P. J.; ZIEBECK, K. R. A. In: Alloys and Compounds of d-Elements with Main Group Elements. Part 2., Landolt-Börnstein, New Series, Group III, v. 19C, p. 75-184, ed. H. R. J. Wijn., Springer, Berlin, 1988. 